EPJ Web of Conferences 66, 06017 (2014)

DOI: $10.1051 /$ epjconf/ 20146606017

(C) Owned by the authors, published by EDP Sciences, 2014

\title{
DVCS at HERMES
}

\author{
Erik Etzelmüller ${ }^{1, a}$ on behalf of the HERMES Collaboration \\ ${ }^{1}$ II. Physikalisches Institut, Justus-Liebig-Universität Gießen, 35394 Gießen, Germany.
}

\begin{abstract}
Azimuthal asymmetries in exclusive electroproduction of real photons by the longitudinally polarized HERA positron beam scattering off an unpolarized hydrogen target $(e p \rightarrow e p \gamma)$ are measured at HERMES. The asymmetries arise from the interference of DVCS with the Bethe-Heitler process, where DVCS provides the theoretically cleanest access to Generalized Parton Distributions. In 2006, HERMES was upgraded with a recoil detector that, together with the forward spectrometer, allowed the detection of all final state particles in the process $e p \rightarrow e p \gamma$. This helped to reduce the background from the associated reaction $e p \rightarrow e \gamma \pi N$ from $12 \%$ down to a negligible level. In addition, the selection and measurement of the associated reaction itself is shown to be feasible and first results have been obtained.
\end{abstract}

\section{Introduction}

The formalism of Generalized Parton Distributions (GPDs) has been extensively studied for the past decade, both theoretically and experimentally [1-3]. They can be measured in hard exclusive reactions such as $e p \rightarrow e \gamma p$. These reactions can be factorized into a hard scattering part and a soft nonperturbative part, with the latter one being described by the leading-twist GPDs $H, \tilde{H}, E$ and $\tilde{E}$. Each of them describes different helicity transitions of the struck quark and/or of the nucleon as a whole.

The GPDs depend on four kinematic variables: the Mandelstam $t=\left(p-p^{\prime}\right)^{2}$, which corresponds to the squared momentum transfer to the nucleon with $p$ and $p^{\prime}$ being the nucleon's initial and final momentum, respectively; the variable $\mathrm{x}$, which is the fraction of the nucleon's longitudinal momentum carried by the struck quark; the skewness $\xi$, which is half the difference of the initial and final fraction of the nucleon's longitudinal momentum carried by the struck quark; and the photon virtuality $Q^{2}$, which is the squared four momentum difference between the incoming and the scattered lepton.

Being hybrids of the nucleon form factors and the parton distribution functions, the GPDs reveal a three-dimensional picture of hadrons by correlating transverse spatial with longitudinal momentum distributions. Furthermore, the second moments of the GPDs $H$ and $E$ are connected to the total angular momentum carried by the quarks, a feature that makes them important for understanding the 'spin puzzle'.

The associated process $\gamma^{*} N \rightarrow \gamma \Delta$ reveals additional GPDs $\left(H_{M}, C_{1}\right.$ and $\left.C_{2}\right)$. These $N \rightarrow \Delta$ GPDs can be connected to the $N \rightarrow N$ GPDs in the large $N_{c}$ limit and would in principle allow flavor decomposition of the latter ones with an estimated accuracy of about $30 \%$ [4].

\footnotetext{
a e-mail: erik.etzelmueller@physik.uni-giessen.de
} 


\section{Deeply Virtual Compton Scattering}

Deeply Virtual Compton Scattering (DVCS) $\gamma^{*} N \rightarrow \gamma N$ is widely referred to as the cleanest process that provides access to GPDs. Unlike the case of the Hard Exclusive Meson Production (HEMP) where the not so well known meson form factors are involved, the real final state photon in DVCS can be considered as a point-like particle. Although the cross section of the final state of a scattered lepton, a recoiling proton and a real photon is highly dominated by the Bethe-Heitler process $(\mathrm{BH})$ at HERMES kinematics, DVCS can be accessed as their interference

$$
|\mathcal{T}|^{2}=\left|\mathcal{T}_{\mathrm{DVCS}}\right|^{2}+\left|\mathcal{T}_{\mathrm{BH}}\right|^{2}+\underbrace{\mathcal{T}_{\mathrm{DVCS}} \mathcal{T}_{\mathrm{BH}}^{*}+\mathcal{T}_{\mathrm{DVCS}}^{*} \mathcal{T}_{\mathrm{BH}}}_{\mathcal{I}},
$$

where the subordinate DVCS amplitude is amplified in the interference term $\mathcal{I}$.

Due to the kinematical prerequisites a direct measurement of the DVCS cross section was not possible at HERMES. However, since the HERMES experiment allowed for different states of beam polarization and charge, as well as target polarization, a whole variety of cross section asymmetries could be obtained where $\left|\mathcal{T}_{\mathrm{BH}}\right|^{2}$ cancels out as well. The cross section can be expanded in a Fourier series with respect to the angle $\phi$, which is the angle between the lepton scattering and the photon production plane. Different Fourier coefficients can be related to different Compton Form Factors (CFFs), which are convolutions of the GPDs with the hard scattering amplitude.

\section{The HERMES Experiment}

HERMES was a fixed target experiment located at the Deutsche Elektronen Synchrotron (DESY) in Hamburg, Germany. It used the 27.6 GeV lepton beam of HERA with either electrons or positrons. Spin rotators at both ends of the experiment provided a longitudinal polarization of the originally transversely polarized beam. The HERMES forward spectrometer consisted of several devices for tracking and particle identification and served as a detector for particles produced with polar angles between 0.04 and $0.22 \mathrm{rad}$. Particles at large polar angles, such as the recoiling proton, could not be detected until the upgrade with the HERMES recoil detector in 2006 [5]. It covered a momentum range of $135-1400 \mathrm{MeV} / \mathrm{c}$ and provided tracking with up to four space points per track using a silicon strip detector and a scintillating fibre tracker.

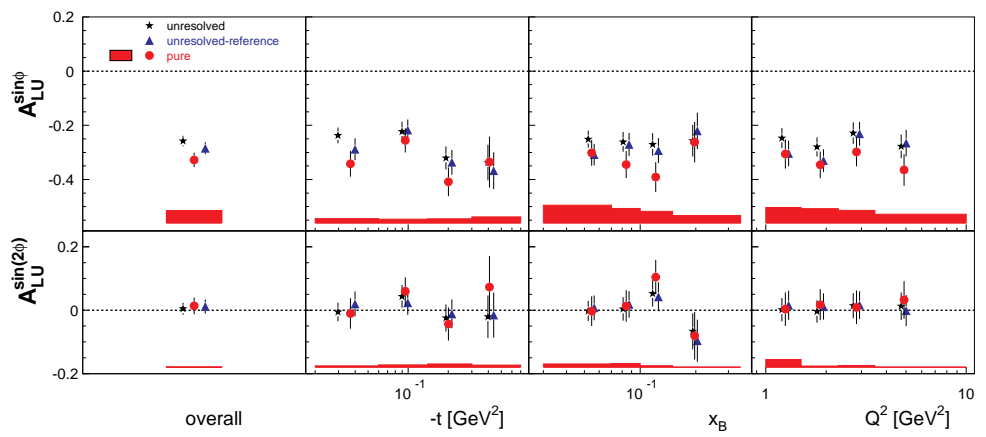

Figure 1. Beam helicity amplitudes of the process $e p \rightarrow e \gamma p$ in projections of $-t, x_{B}$ and $Q^{2}$. Statistical uncertainties are shown by error bars, the red bands represent systematic uncertainties for the 'pure sample'. 


\section{DVCS Results}

Prior to the installation of the recoil detector the exclusiveness of the measurement was ensured by requiring the square of the missing mass to be within an 'exclusive region' around the squared proton mass. This so-called 'unresolved sample' contains background from mainly the associated reaction and Semi-Inclusive Deep Inelastic Scattering (SIDIS) using the HERMES forward spectrometer only. The 'unresolved-reference sample' is defined as a subset of the 'unresolved sample', where kinematics are restricted to be in the acceptance of the recoil detector. The 'pure sample' used the recoil detector in addition and has the same kinematic restrictions as the 'unresolved-reference sample', but is almost free of any background (background contamination is below $0.2 \%$ ). This was achieved by performing a kinematic event fit using all final-state particles including the recoiling proton.

In figure 1 the beam helicity asymmetry amplitudes are presented for these three event samples. The pure sample shows an increase in the magnitude of the leading asymmetry amplitude $A_{L U}^{\sin \phi}$ by $0.054 \pm 0.016$ [6].

An overview of azimuthal asymmetries in the $e p \rightarrow e \gamma p$ reaction measured at HERMES is presented in figure 2. Each asymmetry provides access to a real or imaginary part of the CFFs or to a combination. For example, the amplitudes

$A_{C}$ and $A_{L U}$ are sensitive to the real and imaginary part of the CFF $\mathcal{H}$. Some of the measured asymmetries used a deuterium target and therefore can provide information on DVCS on the neutron.

\section{Associated DVCS}

Prior to the installation of the HERMES recoil detector, the associated reaction $e p \rightarrow e \gamma \pi N$ in the $\Delta$-resonance region constituted the main background for the selection of $e p \rightarrow e \gamma p$ with an estimated average contribution of about $12 \%$. Measurements involving the recoil detector did not only allow a background free measurement of $e p \rightarrow e \gamma p$, but enabled a selection of the associated reaction itself. Asymmetry measurements of the associated reaction not only help to understand the influence of the unresolved background in earlier HERMES DVCS publications but allow access to a new set of GPDs (see section 1). The recoil detector enables the detection of the charged decay particle in the channels $e p \rightarrow e \gamma \pi^{0} p$ and $e p \rightarrow e \gamma \pi^{+} n$. Therefore, the selection of these channels together with rejection of background from $e p \rightarrow e \gamma p$ is possible using kinematic fitting and particle identification in the recoil detector. The purity of about $86 \%$ (76\%) is achieved for the decay channel $e p \rightarrow e \gamma \pi^{0} p\left(e p \rightarrow e \gamma \pi^{+} n\right)$ 
with the main background arising from SIDIS, which is corrected for. In figures 3 and 4 beam helicity asymmetry amplitudes are presented. All leading asymmetries are consistent with zero.

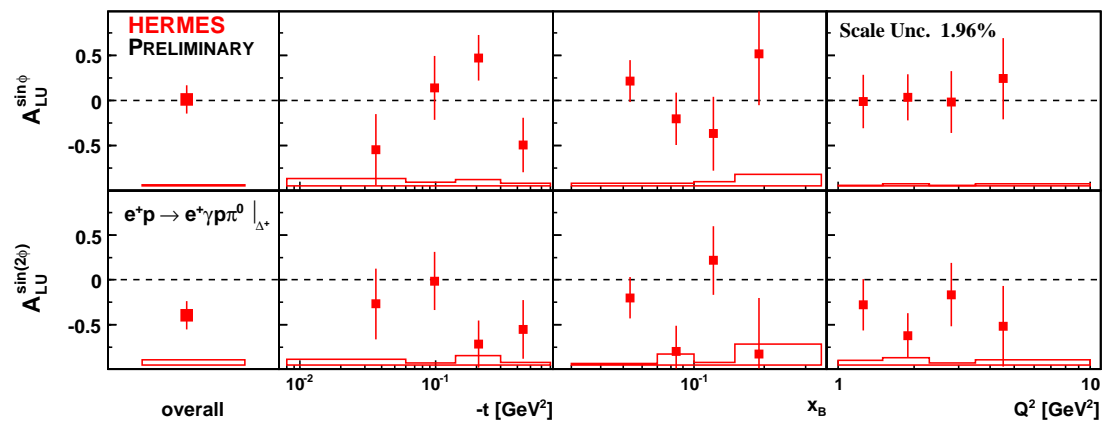

Figure 3. Beam helicity asymmetry amplitudes of the process $e p \rightarrow e \gamma p \pi^{0}$ in projections of $-t, x_{B}$ and $Q^{2}$. Statistical uncertainties are shown by error bars, the red bands represent systematic uncertainties.

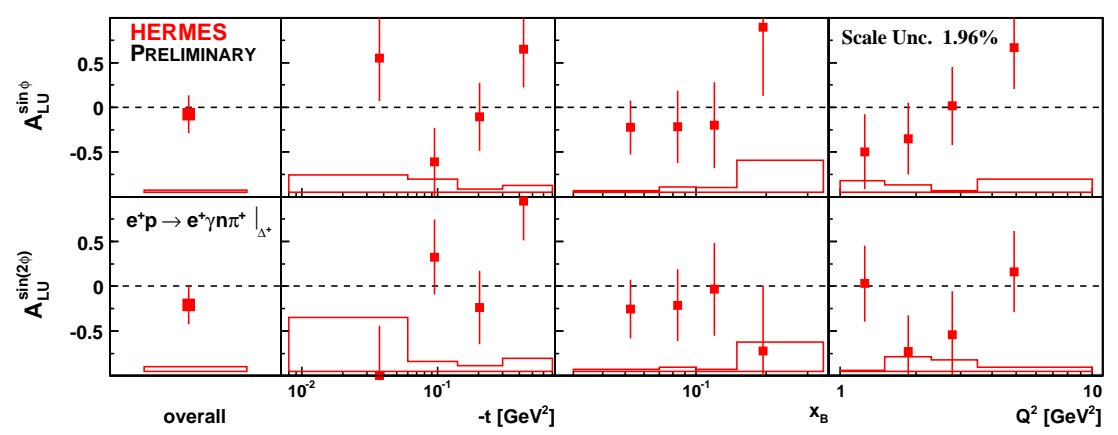

Figure 4. Beam helicity asymmetry amplitudes of the process ep $\rightarrow e \gamma n \pi^{+}$in projections of $-t, x_{B}$ and $Q^{2}$. Statistical uncertainties are shown by error bars, the red bands represent systematic uncertainties.

\section{References}

[1] D. Muller, et al., Fortsch. Phys. 42, 101 (1994)

[2] A. V. Radyushkin, Phys. Lett. B380, 417 (1996), Phys. Rev. D56, 5524 (1997)

[3] X. Ji, Phys. Rev. Lett. 78, 610 (1997), Phys. Rev. D55, 7114 (1997)

[4] P. A. M. Guichon et al., Phys. Rev. D68, 034018 (2003)

[5] A. Airapetian et al., JINST 8, P05012 (2013)

[6] A. Airapetian et al., JHEP 10, 042 (2012)

[7] A. Airapetian et al., JHEP 06, 066 (2008), Nucl. Phys. B829, 1 (2010), JHEP 06, 019 (2010),

Nucl. Phys. B842, 265 (2011), Phys. Lett. B704, 15 (2011), JHEP 07, 032 (2012) 